\title{
GLENOHUMERAL ELEVATION STUDIED IN THREE DIMENSIONS
}

\author{
A. O. BROWNE, P. HOFFMEYER, S. TANAKA, K. N. AN, B. F. MORREY
}

From the Mayo Clinic, Rochester

\begin{abstract}
We studied the position and rotational changes associated with elevation of the glenohumeral joint, using a three-dimensional magnetic-field tracking system on nine fresh cadaveric shoulders. The plane of maximal arm elevation was shown to occur $23^{\circ}$ anterior to the plane of the scapula. Elevation in any plane anterior to the scapula required external humeral rotation, and maximal elevation was associated with approximately $35^{\circ}$ of external humeral rotation. Conversely, internal rotation was necessary for increased elevation posterior to the plane of the scapula. The observed effects of this rotation were to clear the humeral tuberosity from abutting beneath the acromion and to relax the inferior capsular ligamentous constraints.

Measurement of the obligatory humeral rotation required for maximal elevation helps to explain the relationship of the limited elevation seen in adhesive capsulitis and after operations which limit external rotation.
\end{abstract}

In the measurement of shoulder abduction or forward flexion, it is generally accepted that maximal elevation of the arm occurs in the plane of the scapula, but this assumption has never been proven (Braune and Fischer 1888; Johnston 1937; Saha 1973). It is also assumed that maximal arm elevation occurs with external humeral rotation, but again, this relationship has not been quantified (Martin 1932; Davies and Davies 1962). Our investigation was conducted to determine the relationship of glenohumeral elevation to the plane of the scapula and to humeral rotation.

\section{MATERIALS AND METHODS}

We made a three-dimensional kinematic analysis of the movement of the glenohumeral joint on nine fresh cadaveric shoulders, using a 3Space Isotrak system (Polhemus Navigational Sciences Division, McDonnell Douglas Electronics Company, Vermont 05446). We were able to monitor both the three-dimensional position and the orientation of a sensor in relation to a source

A. O. Browne, FRCS I, MCh, Research Fellow P. Hoffmeyer, MD, Visiting Physician

S. Tanaka, MD, Research Fellow

K. N. An, PhD, Professor of Bioengineering

B. F. Morrey, MD, Professor of Orthopaedic Surgery

Biomechanics Laboratory, Department of Orthopaedics, Mayo Clinic/

Mayo Foundation, Rochester, Minnesota 55905, USA.

Correspondence should be sent to Dr K. N. An

(C) 1990 British Editorial Society of Bone and Joint Surgery

$0301-620 X / 90 / 5137 \$ 2.00$

J Bone Joint Surg [Br] 1990; 72-B :843-5. which generated a low frequency magnetic field, as described by An et al (1988). The demonstrated accuracy of measurement of angular rotation is about $1.5^{\circ}$ with a resolution of $0.7^{\circ}$. With the source and the sensor attached to the scapula and the humeral shaft respectively, the relative location and orientation of the humerus with respect to the scapula may be determined. We studied only the orientation of the joint. The Eulerian angle concept was used to resolve the sequence dependency problem of finite rotations.

To define the orientation of the humerus, the first two rotations of the three overall angles may be considered as latitude and longitude on a global representation about the centre of the glenohumeral joint. Latitude then represents the amount of arm elevation, and longitude represents the plane of elevation. The third and final rotation in this Eulerian angle sequence represents axial rotation of the humerus about its shaft.

Nine fresh-frozen cadaveric shoulders were used with careful attention to preservation of the rotator cuff, capsule, and ligamentous structures. To exclude gross pathology, a radiograph was taken of each shoulder before dissection. For reference, a nylon pin was inserted across the humeral shaft at the level of the deltoid tuberosity, perpendicular to the plane formed by the humerus and supinated forearm with the elbow flexed to $90^{\circ}$. The humerus was transected just distal to this pin.

Each specimen was then mounted in an upright anatomical position with the anterior surface of the scapula firmly fixed to a Plexiglas frame with nylon bolts, so as to allow free excursion of the subscapularis with the humerus hanging free. The posterior, infraspinatus 
surface of the scapula was exposed with its vertebral border at $90^{\circ}$ to the horizontal plane. This facilitated digitisation of the medial border and infraspinatus surface of the scapula for subsequent data transformation from the source co-ordinate of the Isotrak system to the scapular co-ordinate of the anatomical system. The humerus was then elevated in 33 planes anterior and posterior to the plane of the scapula under constant load applied at the level of the deltoid tuberosity. In each plane, elevation was recorded through the complete range of internal and external humeral rotation, using a universal joint system. This minimised the influence of the investigator while demonstrating changes in elevation caused by the position of humeral rotation.

\section{RESULTS}

The maximal humeral elevation, related to its angulation to the plane of the scapula, is shown for all nine specimens in Figure 1. The mean plane of maximal elevation was found to occur when the humerus was $23^{\circ}$ anterior to the plane of the scapula (range $10^{\circ}$ to $37^{\circ}, 95 \%$ confidence interval $17^{\circ}$ to $29^{\circ}$ ). Figure 2 shows a typical pattern of elevation, clearly demonstrating the influence of internal and external rotation of the humerus.

Anterior to the plane of the scapula, about $35^{\circ}$ of external humeral rotation was necessary for maximal elevation. Elevation in the scapular plane allowed less arm elevation, and this maximum was seen with less external humeral rotation. Further posterior, there was a plane where external and internal rotation did not influence the maximal elevation. This plane was an average of $31^{\circ}$ posterior to the scapular plane (range $25^{\circ}$ to $40^{\circ}, 95 \%$ confidence interval $28^{\circ}$ to $35^{\circ}$ ). Further posterior to this plane, a mean internal rotation of about $60^{\circ}$ was required to allow maximal elevation.

In all planes anterior to that of the scapula, some external rotation was required to attain maximal elevation, while posterior to the scapula, maximum elevation was coupled with internal rotation. The magnitudes of the external and internal rotation were derived from the sequence of movements as discussed above in the description of Eulerian angle analysis.

\section{DISCUSSION}

Maximal passive elevation of the humerus is possible when it is anterior to the plane of the scapula. Our data have also shown the importance of external rotation when the humerus is anterior to the plane of the scapula, and internal rotation when it is in a posterior plane. We also showed that, anterior to the plane of the scapula, internal rotation or 'derotation' from an externally rotated position reduced the maximal elevation. Similar considerations applied posterior to the scapular plane in relation to the internally rotated position.

Examination of the morphology of the acromion and the coraco-acromial ligament in relation to the mechanism of elevation, show that maximum elevation is impossible in the scapular plane in any rotation, because the acromion blocks maximal excursion. In this plane, humeral rotation causes a rise and fall in elevation as the greater tuberosity rotates under the acromion causing a cam effect. We found a direct relationship, in this plane,

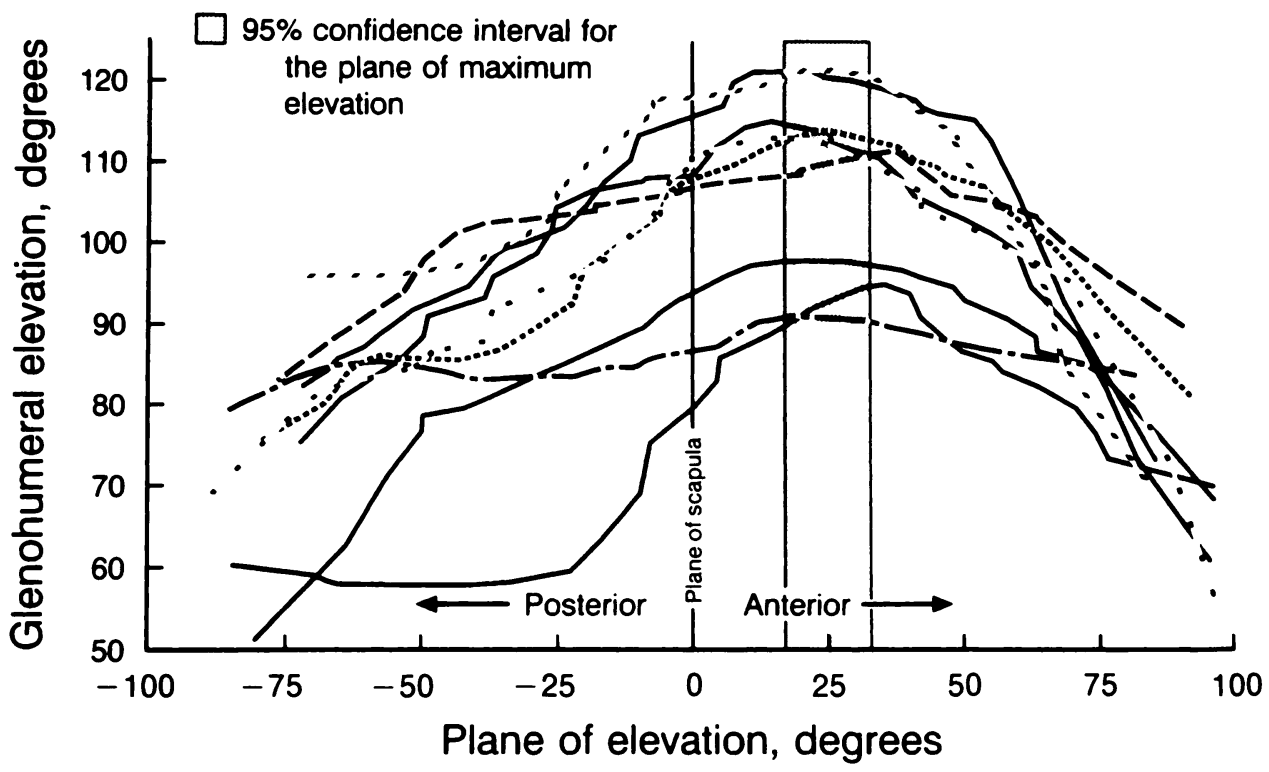

Fig. 1

Maximal elevation of the humerus in various planes, related to that of the scapula. 


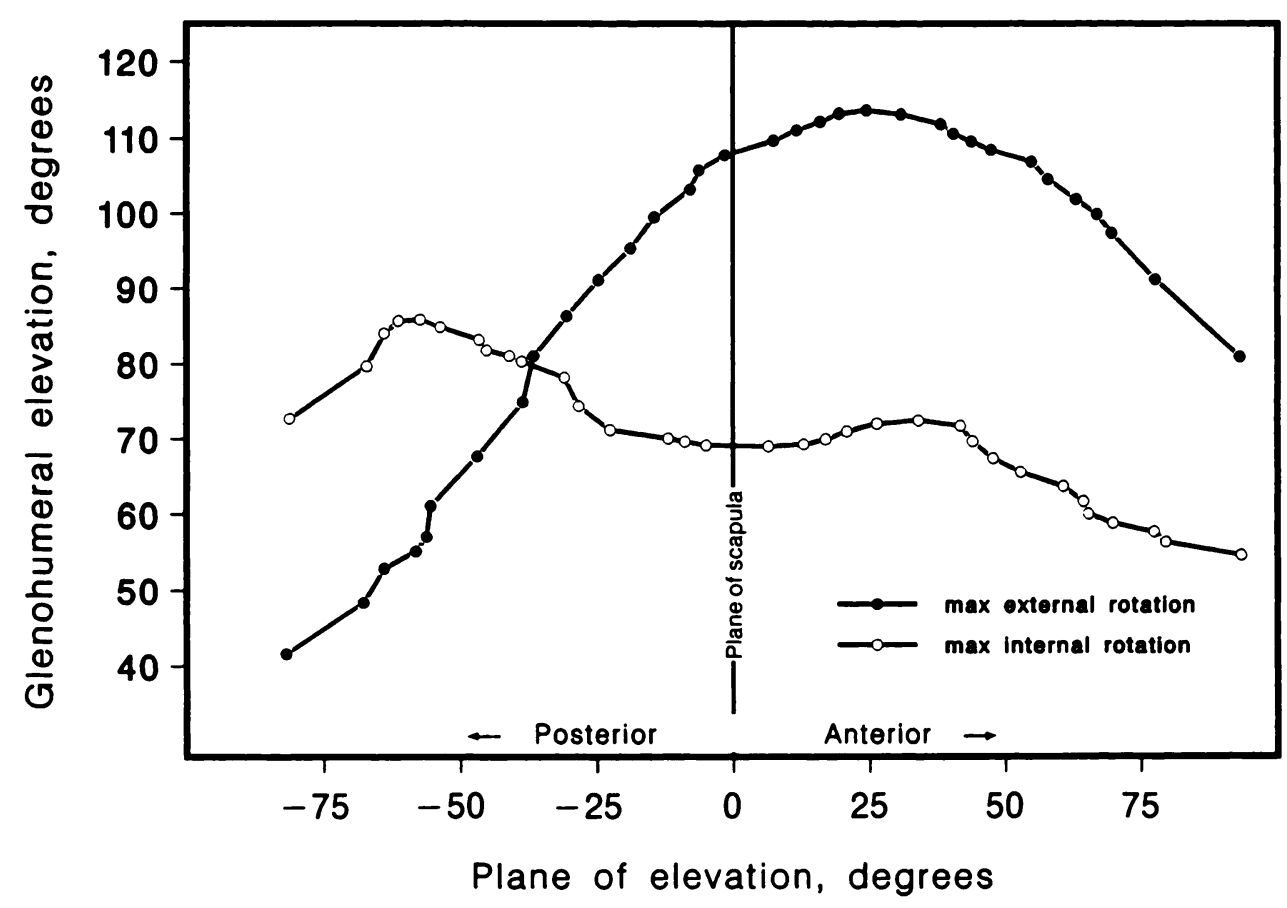

Fig. 2

Typical patterns of maximal humeral elevation in various planes referable to that of the scapula, for the humerus in full internal and full external rotation. The importance of external humeral rotation in planes anterior to that of the scapula and of internal humeral rotation posterior to the scapular plane, is well illustrated.

between the clearing of abutment by the tuberosity and elevation.

When the humerus is anterior to the plane of the scapula, the tuberosity is in the region of the coracoacromial ligament which allows further excursion and greater elevation. External humeral rotation will clear the greater tuberosity from beneath the coraco-acromial arch and also relaxes the capsular ligamentous constraints and allows the maximal elevation seen in this plane (Fig. 2).

The posterior acromion slopes posteriorly, inferiorly, and medially towards the scapular spine. Internal rotation therefore clears tuberosity abutment and allows further elevation in this plane (Fig. 2).

The relationship between clearing abutment and elevation was supported by our observation that elevation increased by an average of $8^{\circ}$ when acromioplasty and resection of the coraco-acromial ligament were performed. The remaining constraints are probably the glenohumeral ligaments; these will be the subject of a future study.

Conclusions. Our study supports the emerging principle of describing glenohumeral movement by 'maximal arm elevation' rather than by maximal flexion or abduction. We have shown the importance of the obligatory humeral rotation.

Contrary to what has been generally accepted, maximal elevation of the glenohumeral joint occurs anterior to the plane of the scapula, and external rotation is required.

Without adequate ranges of external and internal humeral rotation, maximal elevation is impossible either anterior or posterior to the scapular plane, so any attempt at rehabilitation without attention to rotation may be less than satisfactory. Equally, any operation which limits rotation must also decrease maximal elevation.

No benefits in any form have been received or will be received from a commercial party related directly or indirectly to the subject of this article.

\section{REFERENCES}

An KN, Jacobsen MC, Berglund LJ, Chao EY. Application of a magnetic tracking device to kinesiologic studies. J Biomech 1988; $21: 613-20$.

Braune W, Fischer O. Über den Antheil den die einzelnen Gelenke des Schultergürtels an der Beweglichkeit des menschlichen Humerus haben. Abh d Kgl Sachs Ges d Wiss 1888.

Davies DV, Davies F, eds. Gray's anatomy. 33rd ed. London: Longmans, 1962.

Martin CP. A note on the movements of the shoulder-joint. Br J Surg $1932 ; 20: 61-6$

Johnston TB. The movements of shoulder-joint: A plea for the use of the 'plane of scapula' as the plane of reference for movements occurring at the humero-scapular joint. Br J Surg 1937; 25:252-60.

Saha AK. Mechanics of elevation of the glenohumeral joint: its application in rehabilitation of flail shoulder in upper brachial plexus injuries and poliomyelitis and in replacement of the upper humerus by prosthesis. Acta Orthop Scand 1973; 44:668-78. 